\title{
OPTICAL FIBER TIP FABRICATED \\ BY SURFACE TENSION CONTROLLED ETCHING
}

\author{
Pak Kin Wong, Tza-Huei Wang, and Chih-Ming Ho \\ Mechanical \& Aerospace Engineering, University of California, Los Angeles \\ Los Angeles, CA 90095
}

\begin{abstract}
This paper presents a chemical etching method for fabricating probe tips used in scanning near-field optical microscopy. The fiber tip is sharpened by the gradual reduction of the etchant meniscus height that is associated with the decrease of the tip diameter during etching. Control of final tip geometry by adjusting the physical properties of the etching medium is discussed. In addition, we report a novel parallel-etching technique that allows control of the final tip angle by modifying the boundary condition of the meniscus. Tip angle can be adjusted from $7^{\circ}$ to $22^{\circ}$ by simply controlling the gap distance between fibers and number of fibers etched.
\end{abstract}

\section{INTRODUCTION}

Scanning near-field optical microscopy (SNOM) is a promising imaging technique that is not limited by diffraction since it's probe works in close proximity to the surface and images the sample point-by-point with resolution that cannot be achieved by classical optical microscopy. The technique not only allows high spatial resolution but also maintains useful contrast mechanisms, such as fluorescence and polarization, in optical microscopy. After the first demonstration of SNOM in 1984 [1], the technique has been applied to various areas, such as photopatterning of monomolecular films [2] and detection of single fluorescence molecules [3].

As in other forms of scanning probe microscopy, the probe tip is the main factor that determines the quality of the images. The probe tip has to be precisely formed in order to optimize the system performance according to their applications [4]. Presently, the fiber probe tips are fabricated by i) chemical etching in a bulk etchant or at the etchant-solvent interface [5] or ii) a heating and pulling method with a commercial micropipette puller [6]. The heating and pulling method generally produces long tips with small tip angles, which is often undesirable due to low optical transmission [7]. The chemical etching method is undoubtedly the simplest and the most inexpensive optical fiber tip sharpening method, while still allowing for high optical transmission. However, the relationship between the tip angle and etching parameters (e.g. nature of etchant, choice of organic solvent and etching temperature) is not clear, and there is no technique that is both simple and effective that can control the final tip angle over a large range.

In this report, we present a technique based on a theoretical model that can be used to fabricate optical fiber tips with desired geometries. A capillary rise experiment was performed to measure the surface tension and contact angle at the interface to verify the model. Adjustment of the fiber tip angle by controlling the

Travel Support has been generously provided by the Transducers Research Foundation and by the DARPA MEMS and DARPA BioFlips Programs.

physical properties of the etching medium is discussed. Moreover, control of final tip geometry was demonstrated by etching multiple fibers in parallel with different gap distances between fibers and the number of fibers etched at the same time.

\section{PRINCIPLE OF TIP FORMATION}

The typical chemical etching process for fiber tip formation at the interface is shown in figure 1 . The fiber is immersed into a stack solution of etchant and etchant-insoluble organic solvent. The etchant wets the fiber surface with an initial meniscus height. As etching proceeds, the upward pulling force resulting from surface tension decreases due to the reduction of the fiber radius in contact with the etchant. Consequently, the meniscus height reduces progressively until the portion of the fiber below the oil is completely etched, forming the tip.

(a)

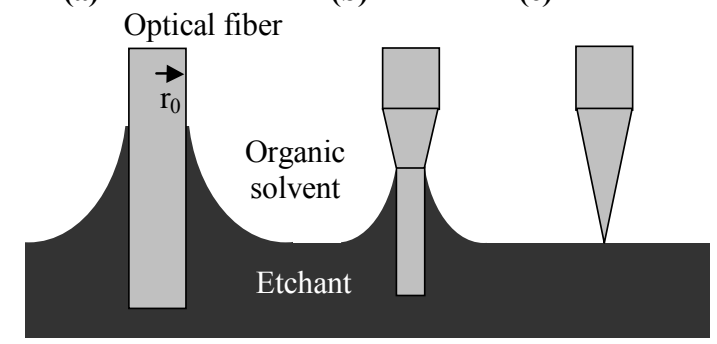

Figure 1. Schematics illustrating the formation of a fiber tip.

Therefore, the final tip profile can be determined according to the relationship between fiber radius and meniscus height, which can be derived by solving the Young-Laplace equation (equation 1 ) in the axisymmetric case with appropriate boundary conditions (equation 2) (figure 2).

$$
\frac{d^{2} y}{d x^{2}}=\left[1+\left(\frac{d y}{d x}\right)^{2}\right]\left\{\frac{\Delta \rho g}{\sigma} y\left[1+\left(\frac{d y}{d x}\right)^{2}\right]^{\frac{1}{2}}-\frac{1}{x} \frac{d y}{d x}\right\}
$$

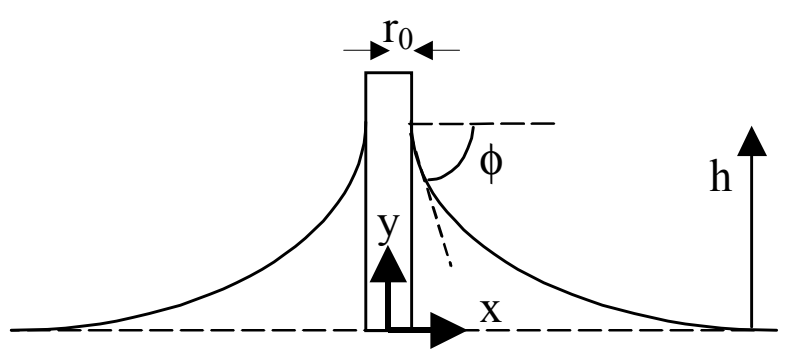

Figure 2. The static meniscus on a circular cylinder immersed into a solution. 


$$
\begin{aligned}
& \frac{d y}{d x}=-\tan \phi \text { at } x=r_{0} \\
& y \rightarrow 0 \text { as } x \rightarrow \infty
\end{aligned}
$$

The equation has been solved both numerically [8] and analytically [9]. Equation 3 shows the first order approximate solution of the meniscus profile solved by the matched asymptotic expansions method [10]. The normalized meniscus height is given by equation 4 :

$$
\begin{gathered}
z(r, \varepsilon)=-c \ln \varepsilon+c(2 \ln 2-\gamma)-c \ln \left[r+\left(r^{2}-c^{2}\right)^{1 / 2}\right]+o(1) \\
z(r=1)=\sin \phi\left\{\ln \frac{4}{\varepsilon(1+\cos \phi)}-\gamma\right\}
\end{gathered}
$$

where $x$ and $y$ are the coordinates, $\Delta \rho$ is the density difference between etchant and solvent, $g$ is gravity, $\sigma$ is surface tension, $\phi$ is the angle between the meniscus and $x$ axis ( $\pi$ - contact angle) $(c=$ $\sin \phi), r_{0}$ is the fiber radius, $z=y / r_{0}$ and $r=x / r_{0}$ are normalized parameters describing the geometry, $l_{0}=(\sigma / \rho g)^{0.5}$ is the capillary length, $\varepsilon=r_{0} / l_{0}$ is the perturbation parameter for solving the equation, and $\gamma$ is the Euler's constant (0.5772). Figure 3 shows meniscus profiles for different fiber radii. The final tip geometry can be estimated by joining the upper ends of the meniscus profiles.

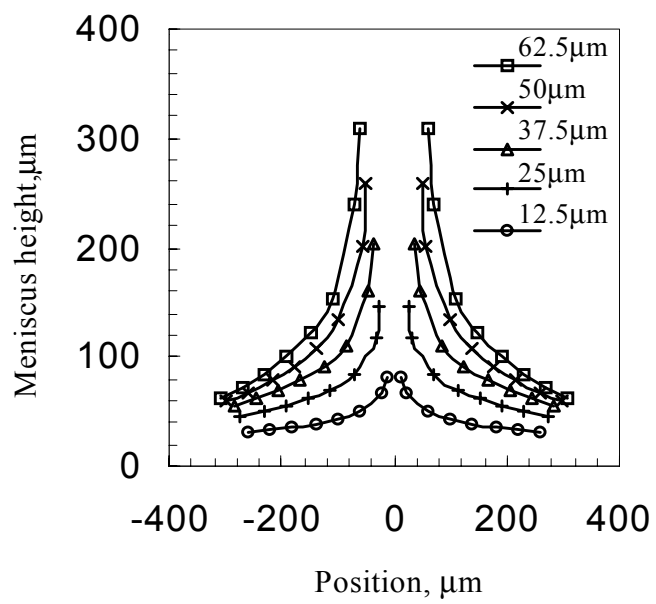

Figure 3. Meniscus profiles for different fiber radii.

\section{TIP ETCHING}

Optical fiber tips were fabricated by etching single mode optical fibers with cladding diameters of $125 \mu \mathrm{m}$ (F-SA, Newport Corporation). Roughly $2 \mathrm{~cm}$ of the polyacrylate coating at the distal end was stripped away and the fiber was cleaned with acetone and DI water. The fiber probe was perpendicularly immersed into the etchant-solvent stack solution. The etchant and organic solvent used in the experiment were hydrofluoric acid (49\%) and silicone oil, respectively. All the etching experiments were carried out at room temperature.

Figures $4 \mathrm{a}$ shows a series of fibers at intermediate stages during etching. The taper region gradually extends as the etching proceeds until formation of the fiber tip. Etching rate of fiber cladding in $49 \% \mathrm{HF}$ can be estimated from figure $4 \mathrm{~b}$, which gives a value of $3.3 \mu \mathrm{m} / \mathrm{min}$. A typical etching process required less than $40 \mathrm{~min}$.

It is clear that the final tip shape is governed by the density difference, surface tension and contact angle at the interface. Density difference can be easily obtained, but the surface tension and contact angle are not available. To verify the model, we measured the surface tension by performing a capillary rise experiment and directly observed the contact angle at the interface (Fig. 5). Surface tension at HF-silicone oil interface and the contact angle were estimated to be $0.047 \mathrm{~N} / \mathrm{m}$ and $17^{\circ}$, respectively. Figure 6 shows the predicted tip profile based on the theoretical model using the aforementioned surface tension and contact angle measured values. Good agreement was obtained when the predicted tip profile was compared to an etched fiber tip.

a)

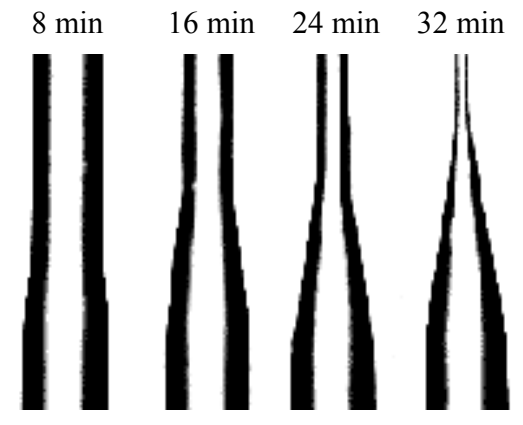

b)

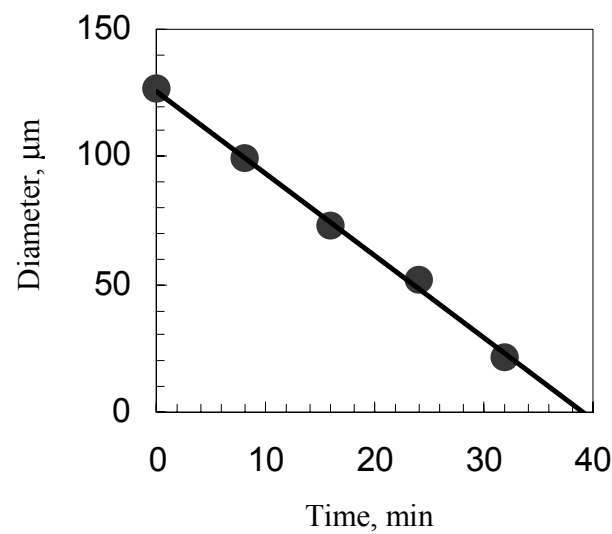

Figure 4. (a) Fibers at intermediate stages during tip formation. (b) Diameter of fiber end as a function of time.

(a)

(b)

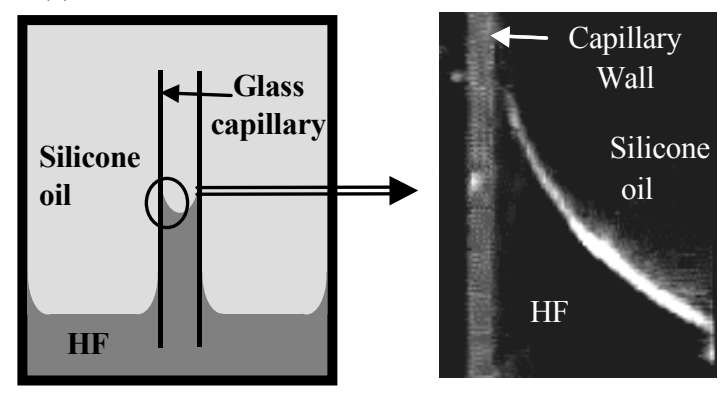

Figure 5. (a) Schematic of the capillary rise experiment. (b) Meniscus inside a glass capillary.

\section{TIP ANGLE CONTROL}

From equation 4 , it is found that a dramatic change in surface tension and the density difference has minimal effect on the meniscus height because of the logarithm in the equation. The physical reason is that the meniscus height is governed by the force balance between the surface tension force and the weight of the etchant fluid entrained along the fiber surface. A slight rise of the meniscus height results in a rapid increase in the volume and 
weight of the etchant fluid that must be balanced by a dramatic rise in surface tension force. Conversely, a small increase in surface tension and density difference has little effect on the meniscus height due to the relationship between height and weight. The meniscus height is sensitive to the change in contact angle at the interface. This is because the contact angle directly affects the meniscus profile and the volume and weight of the etchant. Hence the meniscus height and the final tip profile critically depend on only the contact angle.

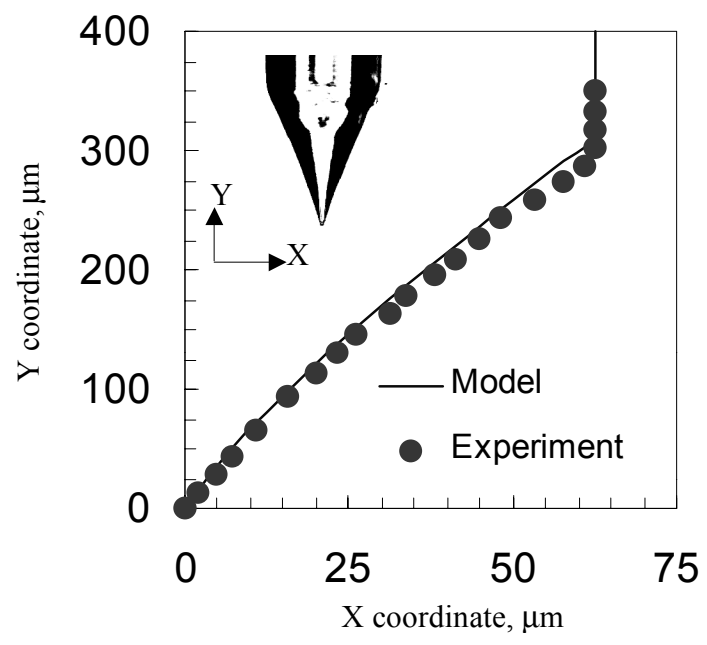

Figure 6. Comparison of tip profiles. - Profile calculated based on the theoretical model with surface tension and contact angle determined in the capillary rise experiment. - Profile measured from an etched fiber tip. Insert: Photography of an optical fiber tip.

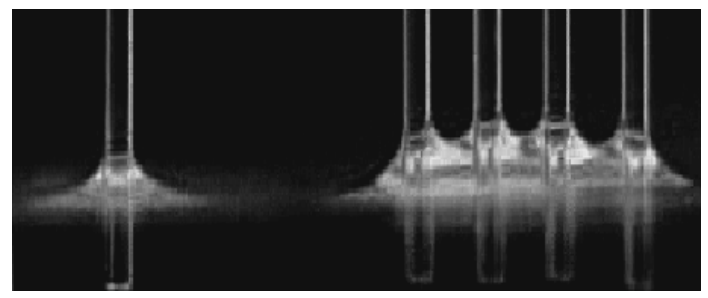

Figure 7. Illustration of meniscus profiles under different boundary conditions.

(a)

(b)

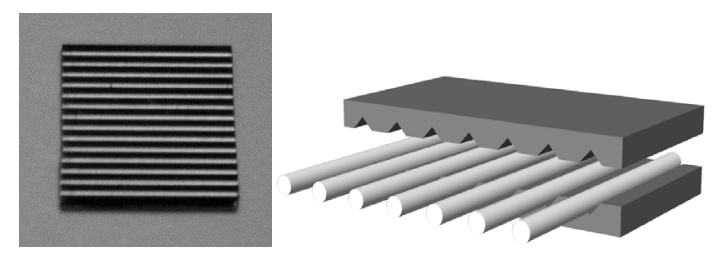

Figure 8. (a) V-groove for parallel-etching experiment. (b) Schematic showing alignment of fibers by v-groove.

Based on the model, optical fiber tips can be fabricated with desired geometries by adjusting the physical parameters that determine the force balance in the system, such as surface tension, density difference of etchant and solvent, and the contact angle of the liquid-liquid interface. These parameters can be altered by changing the organic solvent [5], HF concentration [11], or etching temperature [12]. Replacing the organic solvent allows for a relatively large range of achievable tip angles; however, there is no general rule for the choice of organic solvent to fabricate an optical fiber with specific tip angle. The contact angles at such solvent-etchant interfaces are generally not available. On the other hand, more control of the fiber tip angle can be accomplished by fine-tuning the etchant concentration and etching temperature. To the first order approximation, the change in fiber tip geometry can be estimated by only the change in contact angle. Consequently, one can use the model to predict the fiber tip profile under different etching conditions, such as an increase in etching temperature or etchant concentration. The dependence of surface tension under such conditions is given by thermodynamic theory and the relationship between surface tension and contact angle is related by Young's equation. These relationships can be used to estimate the effect of varying the etching conditions on the final tip geometry.

\section{PARALLEL-ETCHING}

Although our model allows for predictable fabrication of fiber tips, the tip characteristics are sensitive to variations in the experimental conditions. It is valuable if we can have a simple, repeatable and controllable method to fabricate fiber tips with a large range of tip angles. Insight can be obtained by reconsidering the YoungLaplace equation. There are two boundary conditions for the ordinary differential equation. Adjusting the contact angle by changing the etching conditions represents modification of the first boundary condition at the three phase interfacial region. On the other hand, the second boundary condition assumes that the meniscus extends to infinity without interacting with the other boundary. We propose to alter the meniscus profile and meniscus height by introducing a second physical boundary. This opens new opportunity for controlling the tip angle through an easy and efficient method.

Figure 7 illustrates the effect of immersing multiple glass tubes into a solution on the meniscus profile. The meniscus profile was modified and a larger meniscus height was observed. To implement the idea for fiber tip etching, multiple fibers were aligned and etched in parallel. The fibers are positioned with precise gap distances by using v-groove structures etched by $\mathrm{KOH}$ (figure 8).

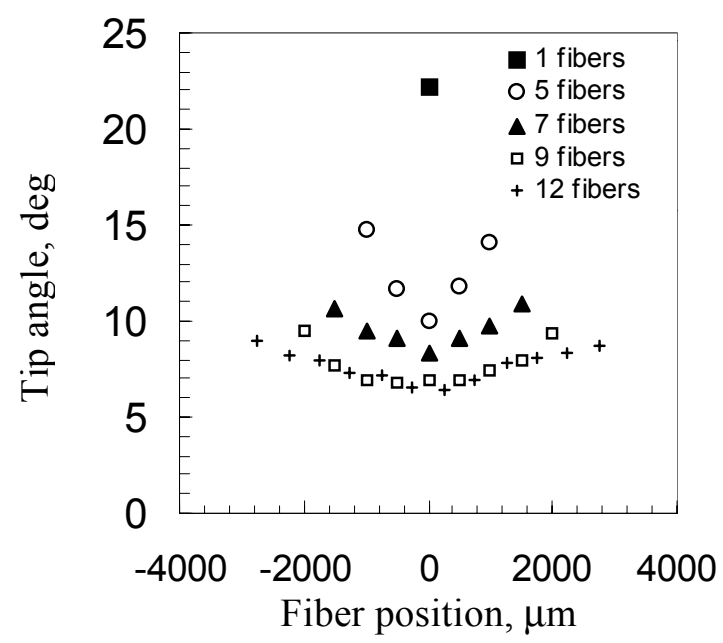

Figure 9. Position dependence of tip angle in paralleletching. 
In parallel-etching, meniscus height is governed by the force balance of surface tension and the weight of the etchant pulled up on the fiber surface. The upward pulling force is proportional to the number of fibers immersed into the stack solution while the weight of the pulled etchant depends on the number of fibers and gap distance. In general, reducing the gap distance and increasing the number of fibers increases the meniscus height. It is also observed that there is a moderate dependence of fiber position on the tip angle (Figure 9). This is because there are only a finite number of fibers immersed in the solution and the meniscuses on the end-most fibers have different boundary conditions than those in between fibers. By using parallel-etching, the cone angle can be adjusted from $7^{\circ}$ to $22^{\circ}$ by simply changing the number of fibers and gap distance (Fig. 10 and 11). Compared to other fiber tip fabrication methods, this novel parallel-etching technique is simple and reproducible.

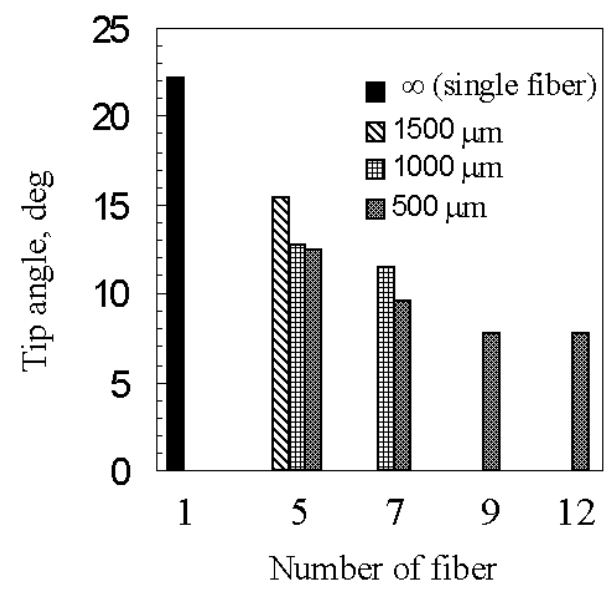

Figure 10. Tip angles of fibers etched under different conditions.

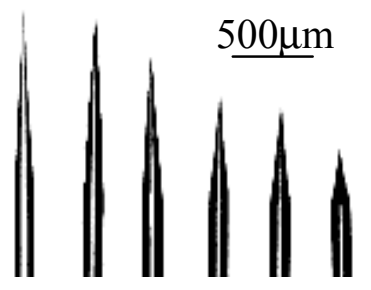

Figure 11. Photographs of optical fiber tip etched under different conditions.

\section{CONCLUSIONS}

A chemical etching technique was developed for fabricating scanning near-field optical microscope probes. The mechanism of the etching process was verified with an independent experiment. We also described a novel technique for controlling the tip angle by etching multiple fibers in parallel. It is foreseeable that the method will extend to other configurations such as array-etching and concentric-etching with other boundary materials. A large selection of materials with different surface properties will generate opportunities for fabricating fiber tips with superior performance for new applications.

\section{ACKNOWLEDGMENTS}

The authors would like to thank Sylvain Masset for his valuable insight and Linda Chun for her technical assistance. This work is supported by DARPA MTO under a Contract N66001-00c-8092 managed by SPAWAR and NSF NIRT program.

\section{REFERENCES}

1. D. W. Pohl, W. Denk, M. Lanz, "Optical Stethoscopy: Image Recording with Resolution $\lambda / 20$ ", Applied Physics Letters, 44, 651 (1984).

2. A. Naber, T. Dziomba, U. C. Fischer, H.-J. Maas, H. Fuchs, "Photopatterning of a Monomolecular Dye Film by Means of Scanning Near-Field Optical Microscopy", Applied Physics A, 70, 227 (2000).

3. E. Betzig, R. J. Chichester, "Single Molecules Observed by Near-Field Scanning Optical Microscopy", Science, 262, 1422 (1993).

4. M. N. Libenson, G. S. Zhdanov, "Optimisation of Near-field Optical Probes", Proceeding of IEEE Lasers and Electro-Optics Society 1996 Annual Meeting, Boston, MA, 11/18-11/96, (1996), pp. 36-37.

5. P. Hoffmann, B. Dutoit, R. Salathé, "Comparison of Mechanically Drawn and Protection Layer Chemically Etched Optical Fiber Tips", Ultramicroscopy, 61, 165 (1995).

6. B.A.F. Puygranier, S. Montgomery, J. Ashe, R.J. Turner, P. Dawson, "Imaging Tip Formation in Single-mode Optical Fibres", Ultramicroscopy, 86, 233 (2001).

7. T. Held, S. Emonin, O. Marti, O. Hollricher, "Method to Produce High-resolution Scanning Near-field Optical Microscope Probes by Beveling Optical Fibers", Review of Scientific Instruments, 71, 3118 (2000).

8. C. Huh, L. E. Scriven, "Shapes of Axisymmetric Fluid Interfaces of Unbounded Extent", Journal of Colloid and Interface Science, 30, 323 (1969).

9. D. F. James, "The Meniscus on the Outside of a Small Circular Cylinder", Journal of Fluid Mechanics, 63, 657 (1974).

10. L. L. Lo. "The Meniscus on a Needle - a Lesson in Matching", Journal of Fluid Mechanics, 132, 65 (1983).

11. A Sayah, C. Philipona, P. Lambelet, M. Pfeffer, F. MarquisWeible, "Fiber Tips for Scanning Near-Field Optical Microscopy Fabricated by Normal and Reverse Etching", Ultramicroscopy, 71, 59 (1998).

12. S.I. Hosain, Y. Lacroute, J. P. Goudonnet, "A Simple LowCost Highly Reproducible Method of Fabricating Optical Fiber Tips for a Photon Scanning Tunneling Microscope", Microwave and Optical Technology Letters, 13, 243 (1996). 